\title{
Gasificación de madera para la obtención de un syngas útil en la producción de biocombustibles y/o productos químicos
}

\author{
Wood gasification to obtain a syngas useful for \\ biofuels and/or chemicals production
}

\section{Syngas de gaseificação de madeira para obter um útil para biocombustíveis e/ou produção de produtos químicos}

\author{
Laura Suárez Hernández ${ }^{1}$; Juan F. Pérez Bayer ${ }^{1 *}$; Rolando Barrera Zapata ${ }^{2^{*}}$ \\ ${ }^{1}$ Grupo de manejo eficiente de la energía (GIMEL), Departamento de ingeniería mecánica, ${ }^{2}$ Grupo CERES, \\ Departamento de ingeniería química, Universidad de Antioquia, Calle 70 No. 52-21, Medellín, Colombia. \\ *rolando.barrera@udea.edu.co
}

Fecha Recepción: 6 de mayo de 2016 Fecha Aceptación: 24 de septiembre de 2016

\begin{abstract}
Resumen
En el presente trabajo se desarrolló un modelo en equilibrio que permite simular el proceso de producción de un syngas útil para la obtención de biocombustibles líquidos y/o productos químicos mediante gasificación en lecho arrastrado. El proceso fue modelado mediante el software Aspen Plus, considerando las etapas de pretratamiento y acondicionamiento de la biomasa (secado, torrefacción y molienda), gasificación en lecho arrastrado, limpieza y acondicionamiento del syngas producido, y ajuste de la relación $\mathrm{H}_{2} / \mathrm{CO}$, adicionalmente se modela la Unidad de Separación de Aire (ASU) para la producción de oxígeno como agente gasificante. La validación del modelo se realizó a partir de datos experimentales reportados en la literatura, mediante el análisis de los errores relativos para las variables de interés: relación $\mathrm{H}_{2} / \mathrm{CO}$, poder calorífico inferior ( $\mathrm{LHV}$, de sus siglas en inglés Lower Heating Value) y eficiencia en frío, obteniendo errores de $7,8 \%, 11,8 \%$ y $8,8 \%$, respectivamente. Adicionalmente, se evaluó la sensibilidad del modelo para predecir el efecto de variables de proceso como la temperatura de torrefacción y la relación equivalente sobre las variables respuesta $\mathrm{H}_{2} / \mathrm{CO}$ y LHV, obteniendo con el modelo tendencias similares a las reportadas en la literatura bajo diferentes condiciones de operación, lo cual muestra que el modelo es sensible a cambios en los parámetros del proceso. Por tanto, se considera que el modelo desarrollado es una herramienta computacional útil para realizar análisis de sensibilidad en procesos de producción de biocombustibles líquidos y/o productos químicos a partir de gasificación de biomasa en lecho arrastrado.
\end{abstract}

Palabras clave: gasificación en lecho arrastrado, Aspen Plus, biocombustibles líquidos, biomasa, syngas.

\section{Abstract}

A model to simulate the entrained flow gasification process to produce a syngas useful in the synthesis of liquid biofuels and/or chemicals was developed. The model is simulated in Aspen Plus and includes the stages of: pretreatment and conditioning of biomass (drying, torrefaction and grinding), entrained flow gasification, cleaning and conditioning of syngas produced, adjustment of $\mathrm{H}_{2} / \mathrm{CO}$ relation, and an Air Separation Unit (ASU) for oxygen production as gasifying agent. The model validation was performed from experimental data reported in the literature, by analyzing the relative errors for the interest variables: $\mathrm{H}_{2} / \mathrm{CO}$, lower heating value and cold gas efficiency, founding errors of $7.8 \%, 11.8 \%$ and $8.8 \%$ respectively. Additionally, the sensibility of the model to predict the effect of process variables as torrefaction temperature and equivalence ratio on response variables, showed similar tendencies to those found 
in the literature. Thus the model is considered a useful computational tool for sensitivity analysis of entrained flow gasification of biomass to produce liquid biofuels and/or chemicals.

Keywords: entrained flow gasification, Aspen Plus, liquid biofuels, biomass, syngas.

\section{Resumo}

Neste trabalho mostra-se um modelo de equilíbrio termodinâmico o qual foi desenvolvido para simular o processo de produção de syngas num gaseificador de leito arrastado, para a obtenção de biocombustíveis líquidos e/ou produtos químicos. O processo foi modelado usando o software Aspen Plus, considerando as etapas de pre-tratamento e acondicionamento da biomassa (secagem, torrefação e moagem), gaseificação em leito arrastado, limpeza e condicionamento do gás de síntese produzido, e a etapa final da reação shift para ajustar a relação $\mathrm{H}_{2} / \mathrm{CO}$. Adicionalmente, também foi modelada uma Unidade de Separação de Ar (ASU) para a produção de oxigênio como agente de gaseificação. A validação do modelo foi realizada a partir dos dados experimentais disponíveis na literatura. Os erros relativos encontrados foram de $7,8 \%, 11,8 \%$ e $8,8 \%$, para as variáveis $\mathrm{H}_{2} / \mathrm{CO}$, eficiência a frio, e poder calorifico, respectivamente. Além disso, a avaliação da sensibilidade do modelo para determinar o efeito de variáveis do processo, temperatura de torrefacção e o fator de oxigênio, mostraram bons resultados. Em consequência, considera-se que o modelo desenvolvido é uma ferramenta útil para a análise computacional da sensibilidade dos processos de produção de combustíveis líquidos e/ou produtos químicos de gaseificação de biomassa em reatores de leito arrastado.

Palabras-chave: gaseificação em leito arrastado, Aspen Plus, biocombustíveis líquidos, biomassa, syngas.

\section{Introducción}

Actualmente los combustibles líquidos son principalmente derivados del petróleo, sin embargo con el fin de evitar un déficit de estos y disminuir los impactos ambientales al tratarse de un combustible fósil, se han creado políticas en todo el mundo que buscan incentivar la obtención de combustibles líquidos a partir de fuentes alternativas viables económica, ambiental y energéticamente [1]. Entre las alternativas, se encuentra el uso de fuentes renovables de energía primaria para producir combustibles líquidos con características similares a los derivados del petróleo. Los combustibles obtenidos a partir del uso de material lignocelulósico o algas son considerados como combustibles renovables de segunda y tercera generación, respectivamente [2].

A través de procesos termoquímicos como la gasificación, es posible transformar diferentes tipos de biomasa en combustibles líquidos o gaseosos con altos rendimientos. Por tal razón, se espera que los biocombustibles de segunda generación, como combustibles líquidos sintetizados a partir de la gasificación de biomasa, sean los principales sustitutos de los combustibles derivados del petróleo [3].
Varios autores han desarrollado modelos para simular el proceso de gasificación en lecho arrastrado de biomasa. Adeyemi y Janajreh [4], desarrollaron un modelo cinético en Aspen Plus para la simulación de gasificación en lecho arrastrado de biomasa y carbón. De acuerdo a sus análisis, los autores observaron que al aumentar el diámetro y la altura del reactor, se incrementa la fracción molar de $\mathrm{H}_{2}$ y $\mathrm{CO}$ del gas producido. Tremel et al. [5] compararon el proceso de gasificación en lecho fluidizado y en lecho arrastrado a diferentes escalas, a partir del desarrollo de un modelo en equilibrio en Aspen Plus para cada una de las tecnologías. Encontraron que ambos reactores presentan resultados similares para la obtención de syngas a una presión $2,5 \mathrm{MPa}$ y que la tecnología de lecho arrastrado puede considerarse como alternativa para la gasificación a pequeña escala. Ramzan et al. [6] desarrollaron un modelo en equilibrio para la gasificación de biomasa mediante el software Aspen Plus. Realizaron análisis de sensibilidad para evaluar el efecto de la temperatura, la relación equivalente, el contenido de humedad de la biomasa y la relación vapor-biomasa en el poder calorífico del syngas, la producción de $\mathrm{H}_{2}$ y la eficiencia en frío (CGE). Encontraron que la temperatura incrementa la producción de $\mathrm{CO}$ y $\mathrm{H}_{2}$, mientras que la relación 
equivalente disminuye la producción de estos (CO y $_{2}$ ) y por tanto la CGE. Por su parte, el contenido de humedad en la biomasa aparentemente afecta el poder calorífico del gas y la inyección de vapor favorece la producción de hidrógeno.

Xiangdong et al. [7] desarrollaron mediante Aspen Plus un modelo en equilibrio de tres etapas para la gasificación en lecho arrastrado de carbón de acuerdo a la tecnología de Texaco. El modelo considera etapas de pirólisis, combustión y gasificación y, según los autores, resulta útil para realizar cálculos de composición del gas, conversión de carbón y temperatura de gasificación. Kunze et al. [8] desarrollaron también un modelo en equilibrio para un gasificador en lecho arrastrado usando Aspen Plus. Con el modelo analizaron el efecto de la tecnología de alimentación (seco y en húmedo) y de refrigeración del syngas (seco y húmedo) sobre la eficiencia del proceso. Concluyen que la alimentación en seco resulta más eficiente con respecto a la alimentación en húmedo (con CGE de $83 \%$ y $72 \%$, respectivamente) y la refrigeración del syngas en húmedo genera mayores pérdidas exergéticas. Adeyemi et al. [9] desarrollaron un modelo numérico para estudiar la gasificación en lecho arrastrado de dos combustibles (carbón Kentucky y residuos de madera), evaluando el efecto de la relación equivalente, la presión y la temperatura en la gasificación. Los autores resaltan que la calidad final del gas (mayor contenido de $\mathrm{H}_{2}$ y $\mathrm{CO}$ ) depende de la materia prima, y además que los incrementos en la temperatura, relación equivalente y presión durante el proceso, aumentan la producción de $\mathrm{CO} \mathrm{y} \mathrm{H}_{2}$ para ambos combustibles.

Otros autores han realizado estudios en los cuales implementan modelos que permiten analizar el proceso de obtención de diferentes productos como gas natural sustituto, $\mathrm{H}_{2}$, productos químicos y/o combustibles líquidos a partir de diferentes tecnologías de gasificación utilizando el software Aspen Plus. Biagini et al. [10] simularon la gasificación de carbón en lecho arrastrado para producción de $\mathrm{H}_{2}$ (de acuerdo a la tecnología Siemens). En su modelo (que utiliza información cinética) incluyen etapas de precalentamiento, devolatilización, combustión, gasificación y enfriamiento. Un aporte interesante de ese estudio es el reporte de la relación oxígeno-carbón $(0,42)$ y vapor-carbón $(0,25)$ que maximizan la producción de $\mathrm{H}_{2}$ a las condiciones estudiadas. Muresan et al. [11] simularon la producción de $\mathrm{H}_{2}$ mediante la gasificación de diferentes mezclas de carbón-biomasa. La tecnología de gasificación simulada fue de lecho arrastrado de acuerdo a la tecnología de Siemens para alimentación en seco. Como dato interesante observaron que al aumentar la proporción de biomasa en la mezcla disminuyen tanto la producción de $\mathrm{H}_{2}$ como la eficiencia energética del proceso. Barrera et al. [12] desarrollaron un modelo para evaluar la producción de Gas Natural Sustituto (SNG) a partir de la gasificación de carbón en lecho arrastrado. Analizaron el uso de diferentes tipos de carbón mediante dos tecnologías de alimentación: seco y húmedo a partir del análisis de eficiencias energéticas, potencia y calidad del SNG obtenido. Concluyeron que el tipo de carbón no afecta significativamente los parámetros energéticos evaluados, mientras la tecnología de alimentación sí tiene un efecto significativo en el proceso. Un aspecto destacable de ese estudio es el reporte detallado de la implementación de cada una de las etapas del modelo en Aspen Plus, incluyendo etapas para la preparación del carbón, gasificación, limpieza del gas, reactor agua-gas y reactor de metanación.

A partir de la literatura consultada, se observa que hay diversos estudios en los que se desarrollan modelos de gasificación en lecho arrastrado con biomasa para evaluar el efecto que tienen algunos parámetros de operación sobre variables como la composición del gas, la eficiencia energética y la eficiencia exergética, así como estudios en los cuales desarrollan modelos que permiten analizar el proceso de obtención de diferentes productos $\left(\mathrm{H}_{2}\right.$, SNG, productos químicos o biocombustibles, entre otros) a partir de gasificación de biomasa. Con respecto a la simulación para la producción de productos químicos y combustibles líquidos mediante gasificación en lecho arrastrado de biomasa, no se encontraron reportes en los que se presente un modelo completo para la producción de un syngas útil en la obtención de productos químicos y combustibles líquidos, que incluya además del proceso de gasificación en lecho arrastrado, etapas de pretratamiento de la biomasa (secado, torrefacción y molienda) y de ajuste de la relación $\mathrm{H}_{2} / \mathrm{CO}$ mediante un reactor agua-gas. Por lo cual, en este trabajo se desarrolla un modelo en equilibrio usando software especializado (Aspen Plus), que permite analizar el efecto de las condiciones de operación del proceso en la obtención de biocombustibles líquidos y/o productos químicos a partir de gasificación en lecho arrastrado. 


\section{Metodología}

Tomando como referencia reportes de la literatura [1-2,12], se definió el diagrama del proceso para la producción de un syngas útil para producir combustibles líquidos y/o productos químicos a partir de la gasificación en lecho arrastrado de biomasa, Figura 1. Para el proceso seleccionado, se desarrolló un modelo en equilibrio termodinámico mediante el software Aspen Plus, el cual se validó a partir de datos experimentales reportados en la literatura. A continuación se describe de manera detallada el proceso, el modelo desarrollado y la estrategia de validación con la cual se verifica la pertinencia del modelo.

\section{Descripción del proceso}

El proceso modelado incluye subprocesos o etapas para: acondicionamiento de biomasa, producción de oxígeno (agente gasificante) mediante una Unidad de Separación de Aire (ASU), gasificación, limpieza/acondicionamiento del syngas y reacción agua-gas para ajustar la relación $\mathrm{H}_{2} / \mathrm{CO}$. El acondicionamiento de la biomasa incluye como etapas principales el secado y la molienda. Adicionalmente, se incorpora un subproceso para evaluar el efecto del pretratamiento de la biomasa (torrefacción) sobre el proceso en general. El diagrama de flujo del proceso (esquema global) se muestra en la Figura 1.

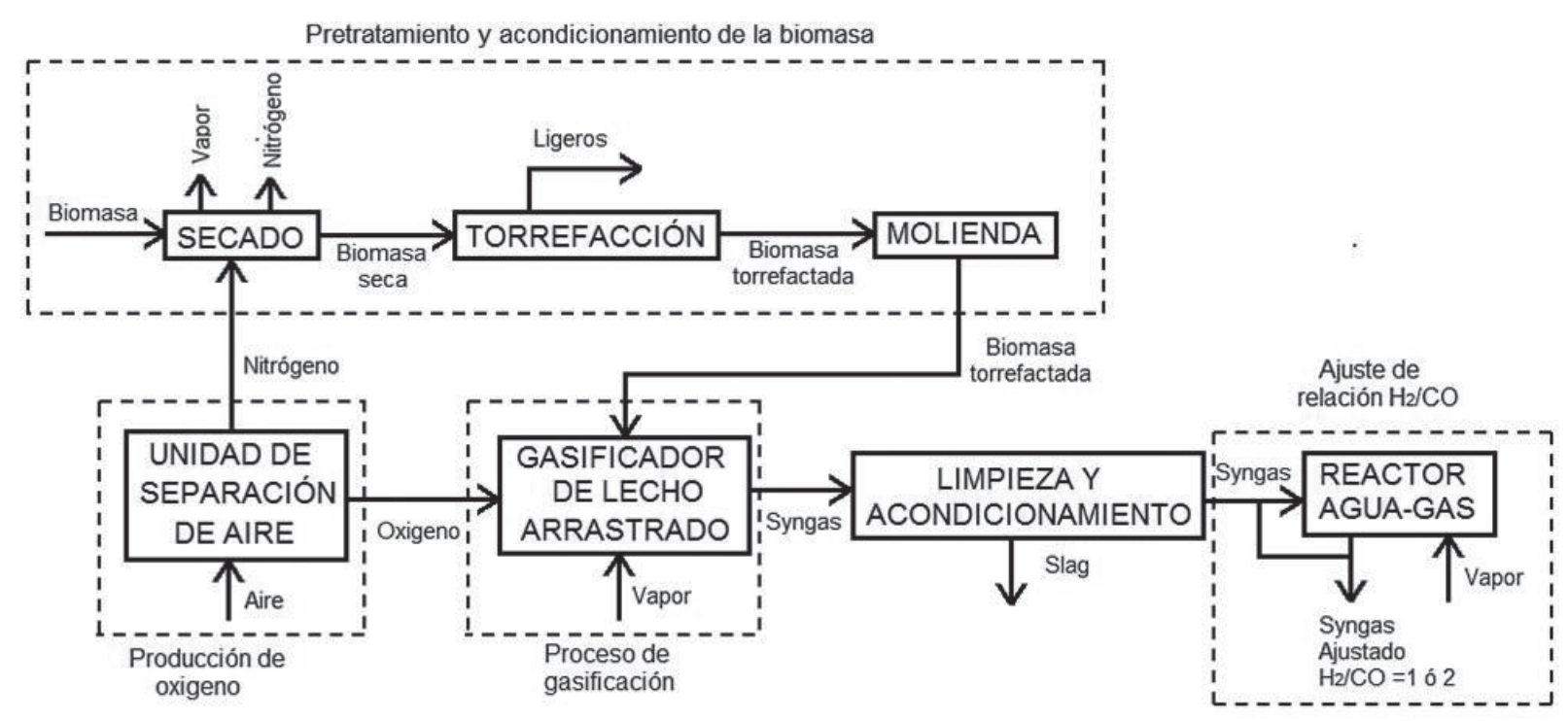

Figura 1. Diagrama de flujo del proceso de gasificación en lecho arrastrado para la obtención de un syngas útil para la producción de combustibles líquidos y productos químicos.

\section{Descripción del modelo}

Se utilizó el software Aspen Plus, un simulador comercial ampliamente utilizado en la industria de procesos químicos, petroquímicos, farmacéuticos y biotecnológicos, entre otros. Actualmente, se ha reportado su aplicación en diferentes sistemas de reacción y separación, así como a plantas de procesos completos, encontrándose buena concordancia entre datos reales y datos determinados a través de las simulaciones en el software [4-7,9,11,13]. El software también permite definir subrutinas específicas en lenguaje FORTRAN o construir migración de datos a Excel que amplía el panorama de posibilidades en la elaboración de simulaciones específicas. Para la estimación de propiedades termodinámicas se hizo uso del modelo termodinámico RedlichKwong-Soave-Boston-Mathias (RKSBM), teniendo en cuenta que varios autores han usado este modelo para simular este tipo procesos [4,13-14]. Entre las principales hipótesis o suposiciones del modelo se tienen: i) el aire que entra a la unidad de separación (ASU) está conformado por una mezcla de nitrógeno y oxígeno $(79: 21 \% \mathrm{v})$ a $25^{\circ} \mathrm{C}$ y $1 \mathrm{~atm}$; ii) el proceso se lleva a cabo en estado estacionario sin pérdidas de calor, ni caídas de presión en los equipos ni en las líneas; iii) el proceso de gasificación es autotérmico y se modela como un reactor en equilibrio de acuerdo a la minimización de la energía libre de Gibbs (R-GIBBS), en el cual los productos formados en el equilibrio son: $\mathrm{CO}_{2}, \mathrm{CO}, \mathrm{H}_{2}, \mathrm{CH}_{4}, \mathrm{H}_{2} \mathrm{O}, \mathrm{C}_{2} \mathrm{H}_{4}$ y 
$\mathrm{C}_{2} \mathrm{H}_{2}$, y iv) el reactor agua-gas (WGSR) consiste en un reactor en equilibrio e isotérmico.

Acondicionamiento y pretratamiento de la biomasa. El subproceso de acondicionamiento incluye etapas de secado y molienda de la biomasa. Para la biomasa pretratada se incluye una etapa adicional de torrefacción antes de ingresar al proceso de molienda. La torrefacción es un pretratamiento que mejora las propiedades de la biomasa como biocombustible sólido [15-17]. Varios autores han simulado mediante Aspen Plus el proceso de secado ya sea de biomasa o de carbón a partir de reactores estequiométricos (R-STOIC) con subrutinas en FORTRAN para definir el proceso de liberación de humedad, seguido de un separador FLASH [4,18-19]. Por tanto, en este trabajo se definió la etapa de secado como se muestra en la Figura 2a, mediante la cual la biomasa (corriente WET-BIOM) se lleva a una humedad $\approx 1 \%$ y a una temperatura de $105^{\circ} \mathrm{C}$ (usando la corriente de nitrógeno $\mathrm{N}_{2}$ de la Unidad de Separación de Aire luego de ser precalentada). El proceso se modeló usando un reactor estequiométrico (bloque DRY-REAC, Figura 2a) con una subrutina en FORTRAN. La corriente que sale del reactor (INDRIER) se lleva a un FLASH (bloque DRY-FLSH, Figura 2a) para separar la biomasa seca que sale por la corriente DRY-BIOM de la corriente gaseosa (EXHAUST) que contiene vapor y nitrógeno.

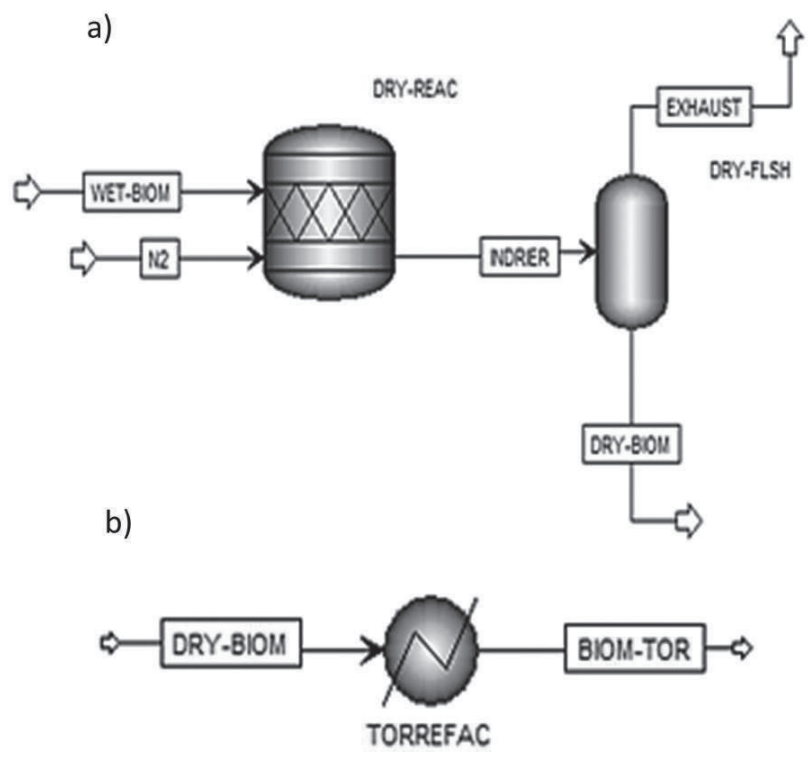

c)

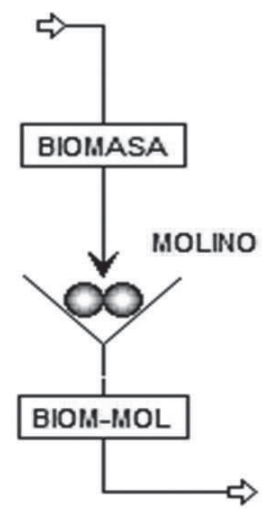

Figura 2. Acondicionamiento y pretratamiento de la biomasa: a) secado; b) torrefacción; c) molienda.

En el proceso de torrefacción, la biomasa libera compuestos volátiles livianos a temperaturas entre 200 y $300^{\circ} \mathrm{C}[17,20]$. De acuerdo a Maski, et al. [21] la energía requerida para el proceso de torrefacción se puede estimar a partir del calor sensible necesario para elevar la temperatura inicial de la biomasa seca hasta la temperatura de torrefacción, asumiendo una eficiencia de torrefacción del 65\%. De esta manera, para modelar este proceso en Aspen Plus, se usó un calentador o HEATER (bloque TORREFAC, Figura $2 b$ ) donde se ingresan los datos de análisis próximo y último de la biomasa a través de la corriente DRY-BIOM, a la temperatura de salida del proceso de secado $\left(105^{\circ} \mathrm{C}\right)$ y sale del subproceso de torrefacción (corriente BIOM-TOR) a la temperatura correspondiente de torrefacción (Figura 2b).

En la molienda se realiza la reducción de tamaño de partícula teniendo en cuenta que la alimentación de combustible a un reactor de lecho arrastrado debe tener un tamaño de partícula menor a $0,1 \mathrm{~mm}$ $[22,23][22,23]$. En este trabajo se utiliza un triturador (bloque MOLINO, Figura 2c), definiendo el tamaño promedio de partícula de la biomasa que ingresa al molino (corriente BIOMASA), la distribución de tamaño de partícula deseado en la corriente de salida (corriente BIOM-MOL) y el índice de triturabilidad de la materia prima (HGI). Este índice de triturabilidad se estima con respecto al contenido de carbono de la biomasa según la correlación reportada por Ibrahim et al. [24]. 
Proceso de gasificación. Se selecciona un gasificador de lecho arrastrado autotérmico, teniendo en cuenta que este tipo de gasificadores no requieren catalizador, presentan baja formación de alquitranes y metano, flexibilidad en la alimentación y disponibilidad comercial a gran escala $[2,3,25]$. La alimentación al gasificador se simula en seco ya que otro tipo de tecnologías, como el alimento en húmedo (slurry) se recomiendan para procesos que incluyen pirólisis donde la producción de bioaceites es importante $[1,5,20,26]$.

Para modelar el proceso de gasificación se pueden utilizar varios tipos de modelos entre los cuales los modelos en equilibrio termodinámico son los más utilizados por su sencillez, capacidad de predicción y bajo tiempo computacional [22,27]. Varios autores han modelado el proceso de gasificación en Aspen Plus a partir de modelos en equilibrio no estequiométricos (minimización de la energía libre de Gibbs), mediante bloques R-GIBBS. Begum, et al. simularon la gasificación de residuos sólidos municipales para un reactor de lecho fijo y validaron con datos experimentales obteniendo errores del $4 \%$ [13]. Kunze y Spliethoff simularon la gasificación de lecho arrastrado de carbón y compararon con datos reales de plantas obteniendo resultados con errores que no superan el $5 \%$ para la predicción de la composición del gas [8]. De igual manera, Xiangdong et al, simularon la gasificación en lecho arrastrado para varios tipos de carbón y validaron los resultados simulados con datos reales de plantas que operan con la tecnología de Texaco, concluyendo que el modelo presentaba buenas predicciones entre los datos simulados y los datos experimentales [7].

Para el software Aspen Plus, la biomasa se considera un compuesto "no convencional", por lo tanto para simular el proceso de gasificación usando los algoritmos y subrutinas incluidas por defecto en el software, es necesario descomponerla en compuestos reactivos (de acuerdo a su análisis próximo y análisis último) antes de ingresarla al gasificador. Para tal efecto se utiliza un reactor de rendimiento (bloque R-YIELD) con ayuda de subrutinas en FORTRAN [12,18]. En la Figura 3 se muestra el modelo utilizado para simular el proceso de gasificación. La biomasa (corriente BIOM$A C$ ) ingresa a un reactor de rendimiento (bloque DESCOMP, Figura 3), donde se descompone en compuestos reactivos según su composición (análisis próximo y análisis último). Estos compuestos reactivos (corriente BIODESC) pasan al bloque GASIF (reactor R-GIBBS) donde reaccionan con el agente oxidante (corrientes VAPOR y OXIGENO) y generan el syngas producto de la gasificación que sale del proceso por la corriente GASIFOUT. La corriente $\mathrm{CO}_{2}$ (Figura 3) simula el flujo del gas de arrastre que impulsa la biomasa a través del proceso. En intensificación de procesos puede considerarse un reciclo de unidades posteriores del proceso, pero en el modelo se presenta como una entrada al sistema. Adicionalmente, para garantizar la conservación de la energía en el proceso, el calor liberado en el bloque DESCOMP (proceso de descomposición de la biomasa) es llevado al gasificador por la corriente $\mathrm{H} 1$.

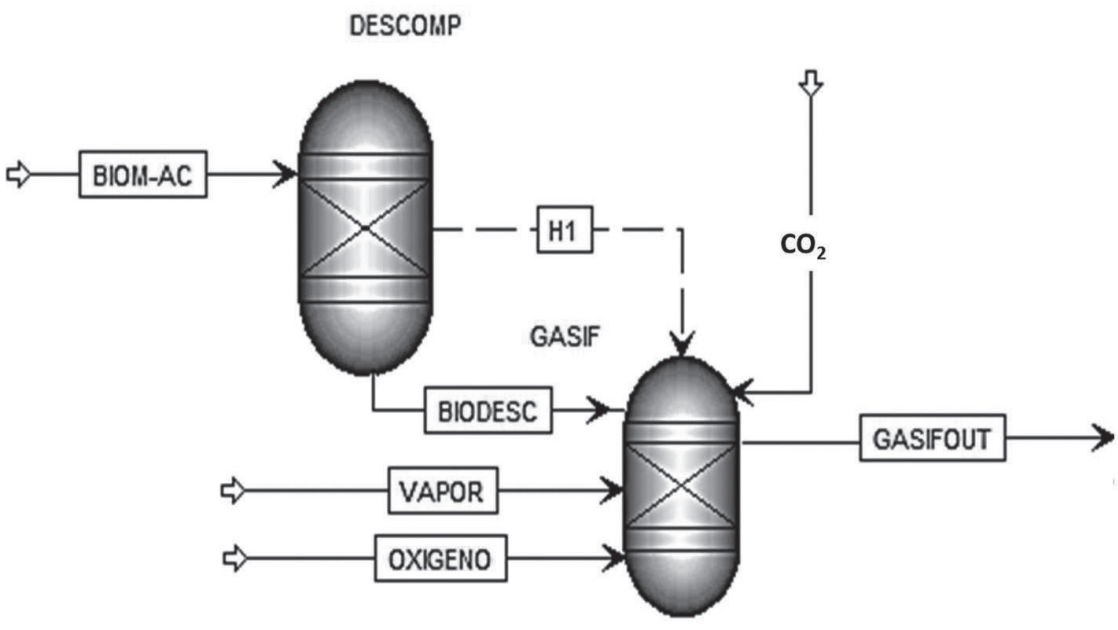

Figura 3. Proceso de gasificación en Aspen Plus. 
Limpiezayacondicionamiento del syngas. Luego de que el syngas sale del gasificador (corriente GASIFOUT), es necesario acondicionarlo para llevarlo al reactor agua-gas. El acondicionamiento comprende el enfriamiento hasta la temperatura de entrada del reactor agua-gas y la separación de compuestos sólidos como escoria y cenizas, los cuales pueden causar ensuciamiento del reactor, desactivación de catalizadores y contaminación de productos [29]. Varios métodos mecánicos como ciclones, filtros de mangas, filtros deflectores, filtros cerámicos, filtros de tela, separadores rotatorios, precipitadores electrostáticos y depuradores de agua se han utilizado para eliminar el material particulado del syngas después de la gasificación [30]. En la presente simulación, la limpieza y el acondicionamiento del syngas se simula pasando la corriente GASIFOUT a través de un calentador o intercambiador de calor (bloque EXCHANG1, Figura 4) donde se enfría el gas hasta el valor determinado. La salida de este intercambiador de calor (corriente COLDGAS, Figura 4) se lleva a un ciclón (bloque SSPLIT), donde se retiran los sólidos por la corriente SLAG y el gas enfriado y limpio se lleva al reactor agua-gas por la corriente GAS, Figura 4.

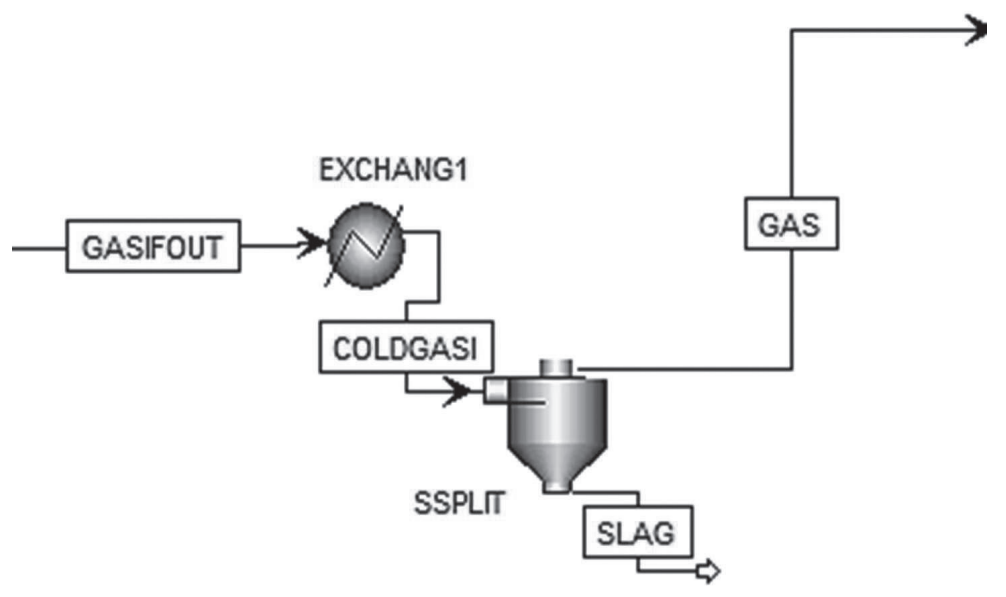

Figura 4. Proceso de limpieza y acondicionamiento del syngas en Aspen Plus.

Reactor agua-gas. En el reactor agua-gas, el syngas reacciona con vapor (reacción (R1)) para ajustar la relación $\mathrm{H}_{2} / \mathrm{CO}$ del syngas al valor deseado (1 para producción de productos químicos, 2 para la producción de combustibles líquidos, 3 para la producción de gas natural sustituto) $[1,12]$. En ocasiones, dependiendo de la relación $\mathrm{H}_{2} / \mathrm{CO}$ del syngas y la relación final deseada, puede ser necesario realizar una derivación (bypass) de la corriente gaseosa antes de que ésta ingrese al reactor agua-gas y luego mezclar la corriente que no ingresó al reactor con la corriente de salida de éste $[1,12,31]$.

$$
\mathrm{H}_{2} \mathrm{O}+\mathrm{CO} \rightarrow \mathrm{H}_{2}+\mathrm{CO}_{2}
$$

El modelo en Aspen Plus utilizado para simular esta etapa del proceso se muestra en la Figura 5. La corriente GAS proveniente de la etapa de limpieza y acondicionamiento se lleva a un divisor de corriente (bloque WGSPLIT, Figura 5) donde se divide la corriente gaseosa en la proporción deseada (de acuerdo a $[1,12,32,33]$, entre un $10 \%$ y un $70 \%$ de la corriente debe pasar por el reactor agua-gas). La corriente WGSRIN se lleva a un reactor de equilibrio (bloque WGSR, Figura 5) donde reacciona con la corriente de vapor STEAM de acuerdo a la reacción (R1), cuyo flujo se determina de acuerdo a la relación $\mathrm{H}_{2} / \mathrm{CO}$ deseada al final del proceso. Según las condiciones de operación del reactor (temperatura y presión) pueden formarse condensados, los cuales se retiran del proceso por la corriente WGSRLIQ, mientras que el producto gaseoso de la reacción (rico en $\mathrm{H}_{2}$ ), se mezcla con la corriente WGSRSPLIT, proveniente del divisor de corriente y que no pasó a través del reactor. Esta mezcla se da en el bloque mezclador WGSRMIX (Figura 5) para generar la corriente ENR-SYNG, que corresponde al syngas con una relación específica $\mathrm{H}_{2} / \mathrm{CO}$. 


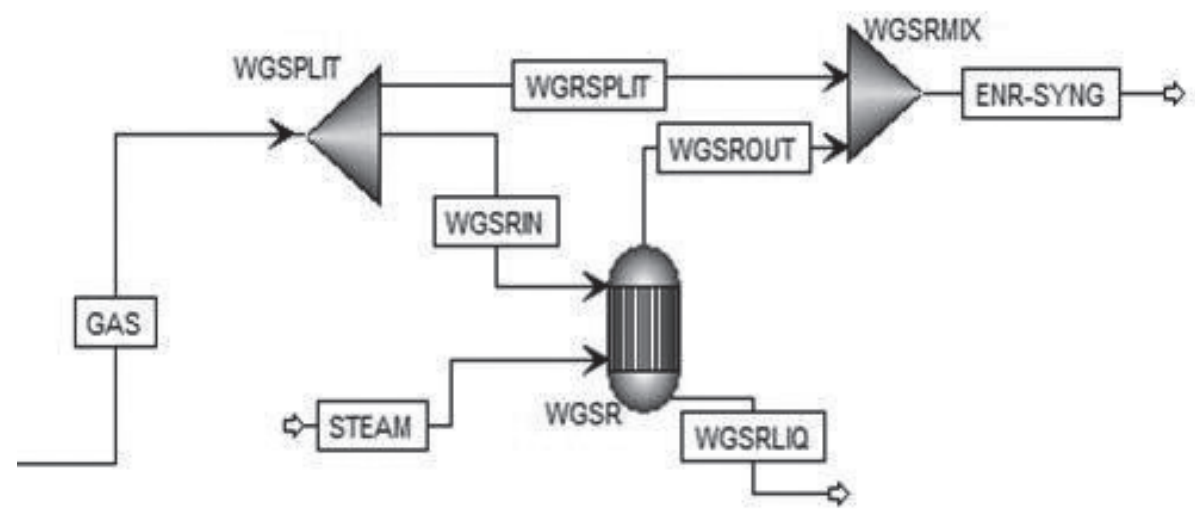

Figura 5. Modelo para el reactor agua-gas en Aspen Plus.

Unidad de Separación de Aire (ASU). Los procesos criogénicos para la separación de aire son considerados como los procesos más eficientes y rentables para la producción de grandes cantidades (escala industrial) de oxígeno y nitrógeno con alta pureza [34-35]. En esta investigación se tomó el modelo planteado por Bose [36] para simular dicho proceso en Aspen Plus. El modelo se basa en la separación criogénica de aire a partir del ciclo simple de Linde-Hampson. En la Figura 6 se muestra el modelo en Aspen Plus. Inicialmente se realiza una compresión isotérmica del aire (corriente AIR) hasta una presión de 30 bar utilizando un compresor (bloque COMPRESS). Con el fin de garantizar que el proceso sea isotérmico, la temperatura del aire comprimido (corriente AIRCOM) se ajusta luego en un enfriador (bloque COOLER 1, Figura 6). Posteriormente, la corriente de salida del enfriador (corriente AIRDCOM) se enfría en un intercambiador de calor (bloque HTEXCH, Figura 6) utilizando como refrigerante la corriente DISTILL proveniente de etapas posteriores del proceso. La corriente de aire comprimido y frío (AIRHX) se lleva hasta $-120^{\circ} \mathrm{C}$ en un segundo enfriador (bloque COOLER2, Figura 6) y posteriormente (corriente ARICMCOL), pasa a través de una válvula de expansión de Joule-Thomson (bloque J-TVALVE) donde alcanza la presión atmosférica y se condensa para generar la corriente de aire líquido "LIQAIR" (Figura 6). Finalmente, la separación del aire líquido en nitrógeno y oxígeno se da en una columna de destilación (bloque DSTCOL). La salida de dicha torre corresponde a la corriente BOTTOM que tiene una composición aproximada de $0.96 \%$ mol de oxígeno que se lleva al proceso de gasificación (Figura 3) y la corriente DISTILL, que tiene una composición aproximada de $0.98 \%$ mol de nitrógeno y se usa como refrigerante en etapas previas del proceso para luego salir del mismo como la corriente DISTHX (Figura 6). Esta corriente, rica en $\mathrm{N}_{2}$, se aprovecha en el proceso de pretratamiento $\mathrm{y}$ acondicionamiento de la biomasa (Figura 2a).

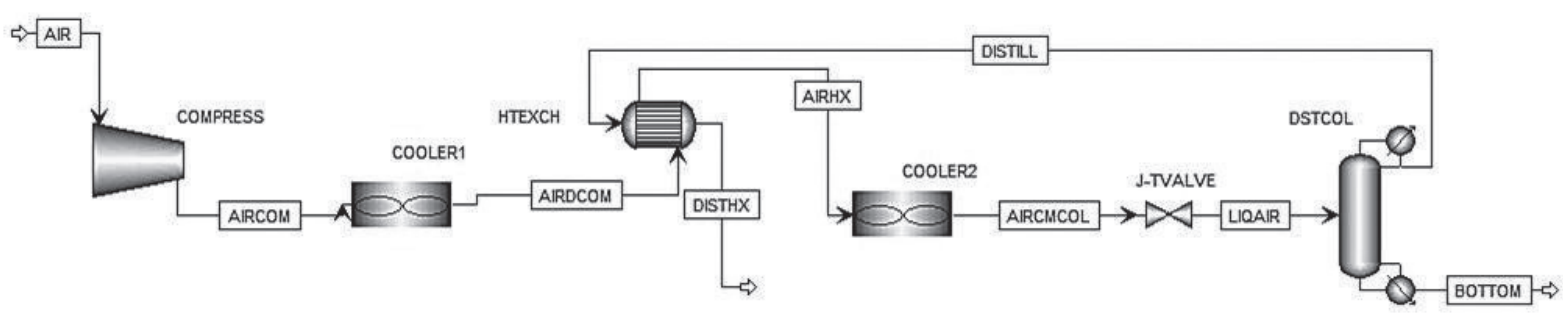

Figura 6. Unidad de separación de aire en Aspen Plus, adaptado de Bose [36]. 


\section{Parámetros del modelo}

Teniendo en cuenta que el modelo desarrollado tiene como objetivo simular la producción de un syngas que posteriormente pueda usarse para la obtención de productos químicos y combustibles líquidos, se definen las siguientes variables respuesta (Ecuaciones 1-4), con el fin de analizar cómo cambian éstas con respecto a los parámetros del proceso.

\section{Eficiencia energética del proceso o eficiencia en frío (CGE)}

$$
C G E=\frac{\dot{m}_{\text {syngas }} * L V H_{\text {syngas }}}{\dot{m}_{\text {biom }} * L H V_{\text {biom }}} * 100
$$

Donde $\dot{m}_{\text {syngas }}$ representa el flujo másico del syngas, $L V H_{\text {syngas }}$ representa el poder calorífico inferior del syngas (valor calculado por el software Aspen Plus), $\dot{m}_{\text {biom }}$ representa el flujo másico de la biomasa y $L V H_{\text {biom }}$ representa el poder calorífico inferior de la biomasa, el cual fue calculado de acuerdo a lo planteado por Ibrahim et al. [24].

\section{Relación $\mathrm{H}_{2} / \mathrm{CO}$}

$$
\mathrm{H}_{2} / \mathrm{CO}=\frac{\text { molesde } \mathrm{H}_{2} \text { enelsyngas }}{\text { molesdeCOenelsyngas }}
$$

\section{Validación del modelo}

Con el fin de evaluar la calidad del modelo con relación a su capacidad de predicción del proceso, se validó tomando como referencia dos trabajos experimentales de gasificación de biomasa en lecho arrastrado reportados en la literatura.

En el primer trabajo seleccionado, reportado por Weiland et al. en 2013 [37], los autores reportan la gasificación de madera (pino y pícea) en una planta piloto de gasificación en lecho arrastrado. Los experimentos se llevaron a cabo a una presión de 2 bar, realizando variaciones de la relación equivalente, $\lambda$ entre 0,43 y 0,5 , usando oxígeno como agente gasificante. La relación equivalente ( $\lambda$ ) está dada por la Ecuación 3 , donde $\dot{m}_{O_{2} \text { real }}$ es el flujo másico de oxígeno alimentado al gasificador y $\dot{m}_{\mathrm{O}_{2} s t q}$ es el flujo másico de oxígeno que se requeriría para que se dé una combustión completa de acuerdo a la estequiometría de la reacción.

$$
\lambda=\frac{\dot{m}_{O_{2} \text { real }}}{\dot{m}_{O_{2} s t q}}
$$

En las Tablas 1 y 2 se encuentran tanto las condiciones de operación como los análisis próximo y último de las biomasas usadas en este estudio.

Tabla 1. Análisis próximo y último para las biomasas usadas en estudio experimental. Adaptado de [37].

\begin{tabular}{lc}
\hline \multicolumn{1}{c}{ Parámetro } & Valor \\
\hline Análisis último base seca (\%wt.) & \\
C (\%) & 51,10 \\
H (\%) & 6,33 \\
N (\%) & 0,00 \\
O (\%) & 42,57 \\
Análisis próximo (\%wt.) & \\
Material volátil (\%) & 80,53 \\
Carbono fijo (\%) & 14,39 \\
Cenizas (\%) & 0,38 \\
Humedad (\%) & 4,70 \\
LHV (MJ/kg) & 19,6 \\
\hline
\end{tabular}

*Valores reportados en base seca, sin cenizas.

Tabla 2. Condiciones de operación usadas en estudio experimental. Adaptado de [37].

\begin{tabular}{lcccc}
\hline \multicolumn{1}{c}{ Parámetro } & Jan-14 & Feb-14 & Feb-16 & Feb-17 \\
\hline Flujo de biomasa $(\mathrm{kg} / \mathrm{h})$ & 40 & 40 & 40 & 40 \\
Flujo de $\mathrm{O}_{2}(\mathrm{~kg} / \mathrm{h})$ & 24,6 & 24 & 27 & 27,5 \\
Flujo de $\mathrm{N}_{2}(\mathrm{~kg} / \mathrm{h})$ & 14,4 & 17,3 & 15,1 & 20,1 \\
Presión-gasificador (bar) & 1,94 & 1,95 & 1,95 & 1,95 \\
\hline
\end{tabular}

*Jan-14, Feb-14, Feb-16, Feb-14: rótulos usados por los autores citados y que hacen relación a las fechas de realización de los experimentos. 
En el segundo trabajo seleccionado, reportado por Weiland en 2014 [38], los autores realizaron la gasificación en lecho arrastrado de 4 combustibles (madera cruda y madera con 3 condiciones de torrefacción: $300^{\circ} \mathrm{C}, 400^{\circ} \mathrm{C}$ y demo). Los experimentos sellevaron a cabo dejando constantes la presión y la relación equivalente, $\lambda$ (Ecuación 3 ), usando oxígeno como agente gasificante. En las
Tablas 3 y 4 se encuentran tanto las condiciones de operación como los análisis próximo y último de las biomasas usadas en este estudio [38], donde "cruda" hace referencia a la biomasa sin torrefactar y Torr-300, Torr-340, Torr-demo hacen referencia a las condiciones de torrefacción reportadas, de acuerdo a la nomenclatura usada por los autores en su trabajo.

Tabla 3. Análisis próximo y último para las biomasas usadas en estudio experimental. Adaptado de [38].

\begin{tabular}{ccccc}
\hline Parámetro & Cruda & Torr-300 & Torr-340 & Torr-demo \\
\hline Análisis último en base seca y sin ceniza (\% wt.) & & & \\
\hline C (\%) & 51,36 & 54,78 & 66,19 & 53,37 \\
H (\%) & 6,26 & 6,04 & 5,36 & 5,93 \\
N (\%) & 0,20 & 0,20 & 0,30 & 0,10 \\
O (\%) & 42,18 & 38,97 & 28,14 & 40,60 \\
\hline Análisis próximo (\% wt.) & & & & \\
\hline Ceniza (\%) & 0,87 & 0,68 & 1,16 & 0,49 \\
Humedad (\%) & 3,0 & 2,4 & 3,1 & 2,2 \\
Volátiles (\%) & 80,03 & 76,42 & 55,04 & 75,01 \\
Carbono Fijo (\%) & 16,10 & 20,50 & 40,70 & 22,30 \\
LHV (MJ/kg) & 19,15 & 20,57 & 25,06 & 20,09 \\
\hline
\end{tabular}

*Valores reportados en base seca. Cruda: Biomasa sin torrefactar; Torr-300, Torr-340, Torr-demo: rótulos usados por los autores citados y que hacen relación a las condiciones (temperatura) de torrefacción.

Tabla 4. Condiciones de operación usadas en estudio experimental. Adaptado de [38].

\begin{tabular}{lcccc}
\hline \multicolumn{1}{c}{ Parámetro } & Cruda & Torr-300 & Torr-340 & Torr-demo \\
\hline Flujo de biomasa $(\mathrm{kg} / \mathrm{h})$ & 55 & 50 & 40 & 50 \\
Flujo de $\mathrm{O}_{2}(\mathrm{~kg} / \mathrm{h})$ & 34 & 34 & 33 & 32 \\
Flujo de $\mathrm{N}_{2}(\mathrm{~kg} / \mathrm{h})$ & 11 & 11 & 10 & 10 \\
Presión - gasificador (bar) & 2 & 2 & 2 & 2 \\
\hline
\end{tabular}

* Cruda: Biomasa sin torrefactar; Torr-300, Torr-340, Torr-demo: rótulos usados por los autores citados y que hacen relación a las condiciones (temperatura) de torrefacción.

Para realizar la validación, se simularon los procesos de gasificación descritos por Weiland et al. en los dos trabajos seleccionados $[37,38]$ ingresando al gasificador la biomasa de acuerdo a la composición reportada por cada uno de los autores y las respectivas condiciones de operación. Posteriormente, se compararon las variables respuesta predichas por el modelo con los datos experimentales reportados para la composición molar del gas $\left(\mathrm{H}_{2}, \mathrm{CO}, \mathrm{CO}_{2}\right.$ y $\left.\mathrm{CH}_{4}\right)$, LHV, relación $\mathrm{H}_{2} / \mathrm{CO}$, CGE y temperatura de torrefacción. La validez del modelo se determinó mediante la Desviación Media Cuadrada (RMSD) y el Error Relativo (RE). Estos parámetros se determinan mediante las Ecuaciones 4 y 5 , que han sido utilizadas para estimar la calidad de los resultados en trabajos de simulación previos [12,39,40].

$$
\begin{aligned}
R M S D & =\sqrt{\frac{\sum_{i=1}^{n}\left(X_{\text {referencia }, i}-X_{\text {Modelo }, i}\right)^{2}}{n}} \\
R E & =\left|\frac{X_{\text {referencia }, i}-X_{\text {Modelo }, i}}{X_{\text {referencia, } i}}\right| * 100
\end{aligned}
$$

Donde $X_{\text {referencia, } i}$ representa los valores experimentales reportados por los autores para cada variable respuesta y $X_{\text {Modelo, }, i}$ representa los 
valores hallados por el modelo para cada variable respuesta y $n$ es el número total de datos.

\section{Resultados de validación}

En la Figura 7 se muestran los resultados de la validación del modelo con respecto a la relación $\mathrm{H}_{2} / \mathrm{CO}$, LHV y CGE. El eje $X$ de la Figura corresponde al valor experimental reportado en la literatura y el eje $Y$ corresponde al valor obtenido mediante simulaciones en el presente estudio. Adicionalmente, en la Tabla 5 se presentan los valores de Error Relativo (RE) y Desviación Media Cuadrada (RMSD) para cada parámetro evaluado. De manera complementaria, la Tabla 6 muestra los resultados comparativos para la composición (\%mol) del syngas simulado y la composición del syngas reportada experimentalmente bajo diferentes condiciones de operación [37,38]. Vale la pena aclarar que los resultados estadísticos de la Tabla 5 se estimaron con base en la información que se presenta en la Tabla 6 y la Figura 7.

A partir de la Figura 7 y los errores calculados en la Tabla 5, se observa que el modelo presenta una muy buena predicción para la relación $\mathrm{H}_{2} / \mathrm{CO}$, debido a que los puntos graficados se encuentran muy cercanos a la línea de $45^{\circ}$ y los valores reportados para RE y RMSD, son bajos (<8\%). De igual manera, se observa que la composición de CO y $\mathrm{H}_{2}$ presentan tanto errores relativos como errores medios cuadráticos bajos, con un valor promedio del RE menor al $10 \%$.

Con respecto a la composición de $\mathrm{CO}_{2}$ y $\mathrm{CH}_{4}$ en el syngas, se observa que hay una baja predicción por parte del modelo para dichos parámetros, con errores relativos del $25,2 \%$ y $100 \%$ (Tabla 5), respectivamente. Weiland et al. [41] obtuvieron resultados similares para el $\mathrm{CH}_{4}$ al desarrollar un modelo de gasificación en equilibrio y compararlo con los resultados experimentales, explicando que en un gasificador real es usual que las concentraciones de $\mathrm{CH}_{4}$ sean mayores a las predichas por un modelo en equilibrio, debido a que los cortos tiempos de residencia no permiten que haya una conversión completa del carbón. Con respecto a esto, es importante tener en cuenta que el orden de magnitud de la fracción molar de $\mathrm{CH}_{4}$ es muy bajo con respecto a los otros compuestos en el syngas ( $<2 \%$ mol, Tabla 6 ), por lo cual se espera que una baja predicción en la composición de este compuesto no represente errores significativos en la estimación o predicción de los parámetros energéticos, tales como poder calorífico y eficiencia de primera ley en frío. a)

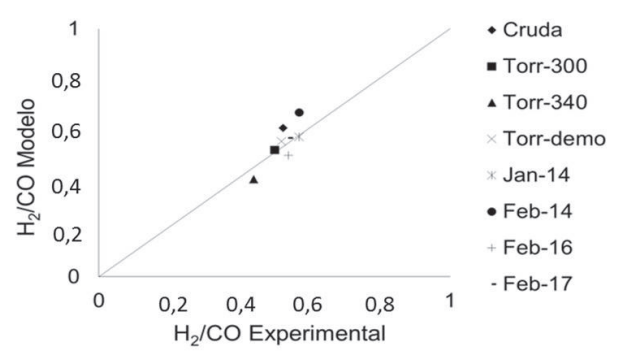

b)

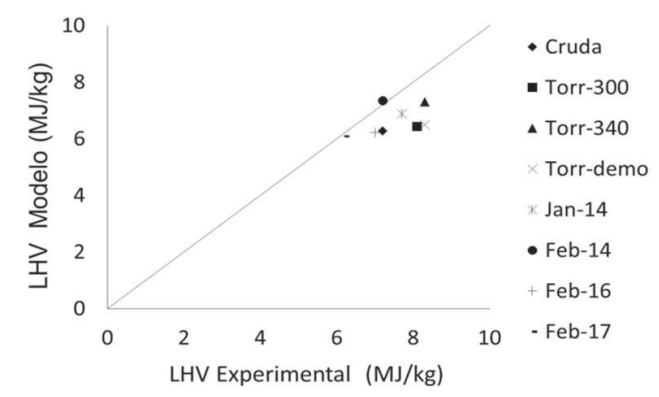

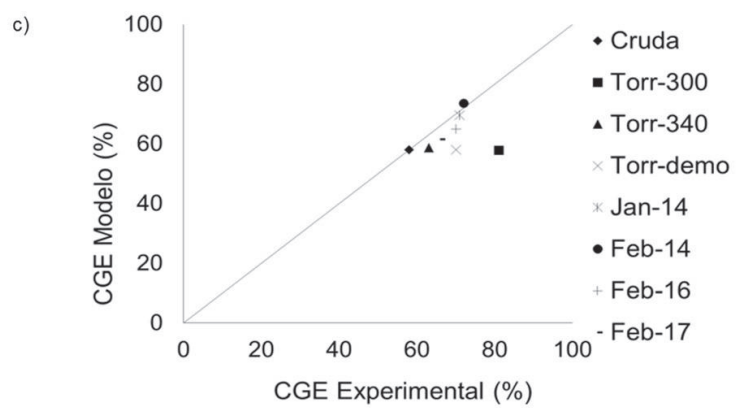

Figura 7. Resultados de validación para: a) Relación $\mathrm{H}_{2} / \mathrm{CO}$ b) LHV, c) CGE. Los rótulos de la leyenda corresponden a los utilizados por los autores citados [37], [38] y hacen relación a las condiciones de operación (temperatura) de torrefacción o a la fecha de realización de sus experimentos. 
Tabla 5. RE y RMSD para validación del modelo.

\begin{tabular}{lcc}
\hline \multicolumn{1}{c}{ Parámetro } & RE (\%) & RMSD \\
\hline $\mathrm{H}_{2}(\% \mathrm{~mol})$ & 10,96 & $\pm 3,63 \% \mathrm{~mol}$ \\
$\mathrm{CO}(\% \mathrm{~mol})$ & 8,49 & $\pm 5,04 \% \mathrm{~mol}$ \\
$\mathrm{CO}_{2}(\% \mathrm{~mol})$ & 25,24 & $\pm 5,94 \% \mathrm{~mol}$ \\
$\mathrm{CH}_{4}(\% \mathrm{~mol})$ & 100 & $\pm 1,74 \% \mathrm{~mol}$ \\
$\mathrm{H}_{2} / \mathrm{CO}$ & 7,84 & $\pm 0,05$ \\
$\mathrm{LHV}(\mathrm{MJ} / \mathrm{kg})$ & 11,77 & $\pm 1,07 \mathrm{MJ} / \mathrm{kg}$ \\
$\mathrm{CGE}(\%)$ & 8,80 & $\pm 9 \%$ \\
$\mathrm{~T}\left({ }^{\circ} \mathrm{C}\right)$ & 9,53 & $\pm 155,17^{\circ} \mathrm{C}$ \\
\hline
\end{tabular}

${ }^{*}$ Calculado como el promedio para las 8 parejas de datos experimentales y simulados.

Con respecto a los parámetros energéticos, LHV (Figura 7b) y CGE (Figura 7c), se observa que el modelo tiende a subestimar estos parámetros.
Pese a esto, el modelo resulta aceptable para su determinación, pues se observa en los puntos graficados que las desviaciones entre los resultados arrojados por el modelo y los valores experimentales son bajas; adicionalmente, en la Tabla 5 se observa que los errores relativos no superan el $12 \%$, y el RMSD asociado al LHV es de $\pm 1,07 \mathrm{MJ} / \mathrm{kg}$, y para la CGE de $\pm 9 \%$.

Con respecto a la estimación de la temperatura alcanzada en el proceso de gasificación (Tabla 5), se observa que la desviación del modelo con respecto a la reportada experimentalmente presenta un error relativo del $9,5 \%$ y que el RMSD para este parámetro es de $\pm 155,17^{\circ} \mathrm{C}$. Estos valores resultan aceptables si se considera el orden de magnitud típico de dicho parámetro en procesos de gasificación en lecho arrastrado $\left(>1000^{\circ} \mathrm{C}\right)$.

Tabla 6. Resultados comparativos para la composición (\%mol) del syngas simulado en este estudio y reportado experimentalmente bajo diferentes condiciones de operación [37,38].

\begin{tabular}{lcccccccc}
\hline Muestra* $^{*}$ & \multicolumn{2}{c}{$\mathbf{H}_{2}$} & \multicolumn{2}{c}{$\mathbf{C O}$} & \multicolumn{2}{c}{$\mathbf{C O}_{2}$} & \multicolumn{2}{c}{$\mathbf{C H}_{4}$} \\
& Sim & Exp & Sim & Exp & Sim & Exp & Sim & Exp \\
\hline cruda [38] & 29,1 & 26,2 & 48,6 & 50,0 & 22,3 & 23,3 & 0,0 & 1,8 \\
Torr-300 [38] & 27,0 & 27,2 & 52,6 & 54,6 & 20,5 & 18,6 & 0,0 & 1,6 \\
Torr-340 [38] & 24,0 & 26,7 & 60,8 & 60,3 & 15,2 & 12,7 & 0,0 & 0,9 \\
Torr-demo [38] & 27,9 & 28,0 & 51,0 & 54,0 & 21,1 & 16,9 & 0,0 & 1,8 \\
Jan-14 [37] & 31,1 & 27,8 & 55,2 & 48,7 & 13,7 & 20,4 & 0,0 & 2,3 \\
Feb-14 [37] & 34,7 & 27,8 & 52,3 & 48,5 & 13,0 & 21,1 & 0,0 & 2,3 \\
Feb-16 [37] & 27,8 & 25,8 & 56,7 & 48,0 & 15,5 & 23,1 & 0,0 & 1,4 \\
Feb-17 [37] & 30,6 & 25,4 & 54,7 & 47,3 & 14,7 & 23,9 & 0,0 & 1,4 \\
\hline
\end{tabular}

* Los rótulos corresponden a los utilizados por los autores citados [37], [38]. Sim=dato simulado; Exp=dato experimental reportado en la literatura

** La composición de $\mathrm{CH}_{4}$ simulado corresponde a trazas del orden de $10^{-5}$, sin embargo por las cifras significativas utilizadas se observa para todos los casos valores de cero.

En las Figuras 8 y 9 se muestran las tendencias (experimentales y simuladas) de la relación $\mathrm{H}_{2} / \mathrm{CO}$ y el LHV del syngas obtenido mediante gasificación de lecho arrastrado, con respecto a la temperatura de torrefacción de la biomasa y a la relación $K$, respectivamente. Se observa tanto en las predicciones del modelo como en los datos experimentales, que al aumentar la temperatura de torrefacción hay una disminución en la relación $\mathrm{H}_{2} / \mathrm{CO}$ y un aumento en el poder calorífico inferior del gas; y que un aumento de $\Lambda$ conlleva a una disminución tanto en la relación $\mathrm{H}_{2} / \mathrm{CO}$ como en el LHV. De lo anterior se concluye que en general el modelo es sensible a los cambios en los parámetros controlables del proceso (condiciones de torrefacción de la biomasa y la relación $\Lambda$ ), cuya capacidad de respuesta se ve reflejada en las tendencias de importantes variables respuesta, tales como $\mathrm{H}_{2} / \mathrm{CO}$ y LHV. Por tanto, es posible destacar que el modelo predice correctamente la tendencia o el comportamiento de las variables analizadas. Esto corrobora que el modelo desarrollado es adecuado para simular el proceso de producción de un syngas útil para la obtención de biocombustibles líquidos y/o productos químicos mediante gasificación de biomasa en lecho arrastrado. 

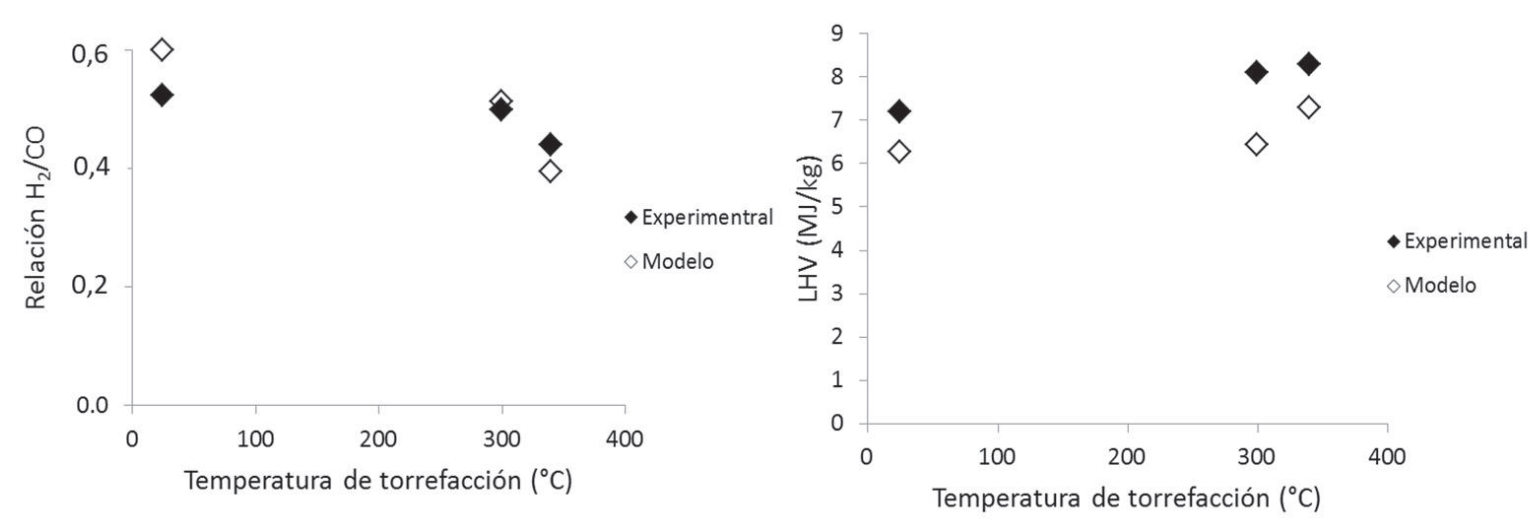

Figura 8. Tendencia de a) relación $\mathrm{H}_{2} / \mathrm{CO}$ del syngas, b) LHV del syngas con respecto a la temperatura de torrefacción. Datos experimentales adaptados de [38].
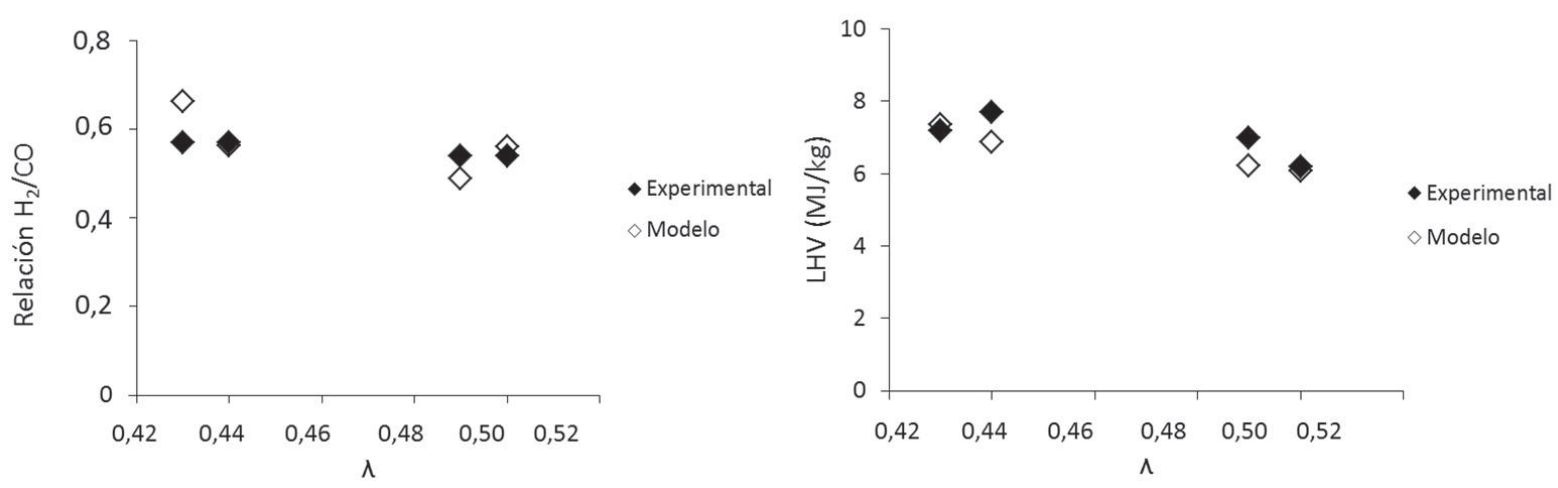

Figura 9. Tendencia de a) relación $\mathrm{H}_{2} / \mathrm{CO}$, b) LHV con respecto a $K$. Datos experimentales adaptados de [37].

\section{Conclusiones}

En el presente trabajo se desarrolló un modelo en equilibrio en Aspen Plus, útil para simular el proceso de producción de biocombustibles líquidos y/o productos químicos mediante gasificación de biomasa en lecho arrastrado. El modelo se validó a partir de datos experimentales reportados en la literatura. De la validación realizada, se puede concluir que el modelo predice satisfactoriamente parámetros de interés como eficiencia en frío del proceso (CGE), relación molar $\mathrm{H}_{2} / \mathrm{CO}$ y poder calórico inferior del syngas (LHV); además de ser sensible a los cambios en los parámetros de entrada como la temperatura de torrefacción y la relación equivalente, $K$. En cuanto a la capacidad de predicción de la composición molar del gas, resulta acertado para la estimación de la cantidad de $\mathrm{H}_{2}$, y $\mathrm{CO}$, aunque para la estimación de la cantidad de $\mathrm{CO}_{2}$ y $\mathrm{CH}_{4}$, las diferencias con respecto a los datos experimentales reportados son significativas. No obstante, tales diferencias no afectan la estimación de parámetros termodinámicos como la eficiencia en frío. Por lo tanto, se puede considerar el modelo como una herramienta útil para simular los procesos de producción de biocombustibles líquidos y/o productos químicos mediante gasificación de biomasa en lecho arrastrado.

\section{Referencias bibliográficas}

[1] Trippe F, Fröhling M, Schultmann F, Stahl R, Henrich E. Techno-economic assessment of gasification as a process step within biomassto-liquid (BtL) fuel and chemicals production. Fuel Process. Technol. 2011;92(11):2169-84.

[2] Swanson R, Platon A, Satrio J, Brown R. Techno-economic analysis of biomass-toliquids production based on gasification. Fuel. 2010;89(Suppl.1):S11-S19.

[3] Ogi T, Nakanishi M, Fukuda Y, Matsumoto K. Gasification of oil palm residues (empty fruit bunch) in an entrained-flow gasifier. Fuel. 2013;104:28-35. 
[4] Adeyemi I, Janajreh I. Modeling of the entrained flow gasification: Kinetics-based ASPEN Plus model. Renew. Energy.2014;82:77-84.

[5] Tremel A, Becherer D, Fendt S, Gaderer M, Spliethoff $\mathrm{H}$. Performance of entrained flow and fluidised bed biomass gasifiers on different scales. Energy Convers. Manag. 2013;69:95106.

[6] Ramzan N, Ashraf A, Naveed S, Malik A. Simulation of hybrid biomass gasification using Aspen plus: A comparative performance analysis for food, municipal solid and poultry waste. Biomass Bioenergy. 2011;35(9):39629.

[7] Kong X, Zhong W, Du W, Qian F. Three stage equilibrium model for coal gasification in entrained flow gasifiers based on aspen plus. Chinese J. Chem. Eng. 2013;21(1):79-84.

[8] Kunze C, Spliethoff H. Modelling, comparison and operation experiences of entrained flow gasifier. Energy Convers. Manag. 2011;52(5):2135-41.

[9] Adeyemi I, Arink T, Janajreh I. Numerical Modeling of the Entrained Flow Gasification (EFG) of Kentucky Coal and Biomass. Energy Procedia. 2015;75:232-9.

[10] Biagini E, Bardi A, Pannocchia G, Tognotti L. Development of an Entrained Flow Gasifier Model for Process Optimization Study. Ind. Eng. Chem. Res. 2009;48(19):9028-33.

[11] Muresan M, Cormos C, Agachi P. Technoeconomical assessment of coal and biomass gasification-based hydrogen production supply chain system. Chem. Eng. Res. Des. 2013;91(8):1527-41.

[12] Barrera R, Salazar C, Pérez J. Thermochemical Equilibrium Model of Synthetic Natural Gas Production from Coal Gasification Using Aspen Plus. International Journal of Chemical Engineering. 2014;1-18.

[13]Begum S, Rasul M, Akbar D. A Numerical Investigation of Municipal Solid Waste Gasification Using Aspen Plus. Procedia Eng. 2014;90:710-7.

[14] Lee H, Choi S, Paek M. A simple process modelling for a dry-feeding entrained bed coal gasifier. Proc. Inst. Mech. Eng. Part A J. Power Energy. 2011;225(1):74-84.

[15] Chen W, Chen C, Hung C, Shen C, Hsu H. A comparison of gasification phenomena among raw biomass, torrefied biomass and coal in an entrained-flow reactor. Appl. Energy. 2013;112:421-30.
[16]Luque R, Campelo J, Clark J. Handbook of Biofuels Production. Woodhead Publishing Series in Energy 2011.

[17] der Stelt M, Gerhauser H; Kiel J and Ptasinski $\mathrm{K}$. Biomass upgrading by torrefaction for the production of biofuels: A review, Biomass and Bioenergy. 2011;35(9):3748-62.

[18] Li J, Zhang X, Pawlak-Kruczek H, Yang W, Kruczek $\mathrm{P}$ and Blasiak W. Process simulation of co-firing torrefied biomass in a 220MWe coalfired power plant, Energy Convers. Manag. 2014,84:503-11,

[19] Aspentech, Aspen Plus Getting Started Modeling Processes with Electrolytes, 2007.

[20] Svoboda K, Pohořelý $M$, Hartman $M$, and Martinec J. Pretreatment and feeding of biomass for pressurized entrained flow gasification. Fuel Process. Technol. 2009;90 (5):629-35.

[21] Maski D, Darr M and Anex R. Torrefaction of cellulosic biomass upgrading. Energy and cost model, Am. Soc. Agric. Biol. Eng. Annu. Int. Meet. 2010, ASABE 2010;6:4443-60.

[22] Luque J, Campelo R, Clark J. Handbook of biofuels production: Processes and technologies. United Kingdom: Woodhead Publishing; 2011.

[23] Desideri E, Manfrida U, Sciubba G. ECOS 2012: The 25th international conference on efficiency, cost, optimization and simulation of energy, 2012.

[24] Ibrahim R, Darvell L, Jones and Williams A. Physicochemical characterisation of torrefied biomass J. Anal. Appl. Pyrolysis. 2013;103: 21-30.

[25] Higman C and van der Burgt M. Gasification Processes Gasification. 2003;85-170.

[26] Santo U, Seifert H, Kolb T, Krebs L, Kuhn D, Wiemer H, Pantouflas E, Zarzalis N. Conversion of biomass based slurry in an entrained flow gasifier. Chem. Eng. Technol. 2007;30(7):9679.

[27] Basu P. Biomass gasification and pyrolysis: practical design and theory. United States: Elsevier Inc., 2010.

[28] Tapasvi D, Kempegowda R, Tran K, Skreiberg $\mathrm{O}$, Grønli M. A simulation study on the torrefied biomass gasification. Energy Convers. Manag. 2015;90:446-57.

[29]Zhang W. Automotive fuels from biomass via gasification. Fuel Process. Technol. 2010;91 (8):866-76.

[30] Wanga M, Wellerb L, Jones C, Hanna D. Contemporary issues in thermal gasification of 
biomass and its application to electricity and fuel production. Biomass and Bioenergy. 2008; 32:573-81.

[31] Twigg M. Chapter 6: Water-gas-Shift Reaction, in Catalyst Handboo. London: M. Publishing, Editor; 1996.

[32] Huisman G, Brinkert J, Cornelissen R. Clean Hydrogen-rich Synthesis Gas Mass and Energy Balance for the Whole Plant. 2009.

[33] Edward P, Abbott J, (12) United States Patent, Vol. 2, no. 12, 2014.

[34] Smith A, Klosek J. A review of air separation technologies and their integration with energy conversion processes Fuel Process. Technol. 2001;70(2): 115-34.

[35] Aneke M, Wang M. Potential for improving the energy efficiency of cryogenic air separation unit (ASU) using binary heat recovery cycles. Appl. Therm. Eng. 2015;81:223-31.

[36]Bose A. Simulation of Air Liquefaction Using Aspen Plus Fulfillment of the Requirement for the, National institute of technology, Rourkela,
India, 2012.

[37] Weiland F, Hedman H,Marklund M, Wiinikka H, Öhrman O, Gebart R. Pressurized oxygen blown entrained-flow gasification of wood powder. Energy and Fuels. 2013;27(2):932-41.

[38] Weiland F, Nordwaeger M, Olofsson I, Wiinikka $\mathrm{H}$, Nordin A. Entrained flow gasification of torrefied wood residues. Fuel Process. Technol. 2014;125:51-8.

[39] Jarungthammachote S, Dutta A. Thermodynamic equilibrium model and second law analysis of a downdraft waste gasifier. Energy. 2007;32:1660-9.

[40] Vaezi M, Passandideh-Fard M, Moghiman M, Chamchi M. On a methodology for selecting biomass materials for gasification purposes. Fuel Process. Technol. 2012;98:74-81.

[41] Weiland $F$, Wiinikka $H$, Hedman $H$, Wennebro J, Pettersson E, Gebart R. Influence of process parameters on the performance of an oxygen blown entrained flow biomass gasifier. Fuel. 2015;153:510-9. 